\title{
REACTION OF NITRILIMINES WITH SUBSTITUTED HYDRAZINES: SYNTHESIS OF 1,2,4,5-TETRAAZA-3-PENTENES AND FORMAZANS
}

\section{Hany M. M. Dalloul}

Department of Chemistry, Faculty of Applied Science

Al-Aqsa University of Gaza

P.O.Box 4051, Gaza 76888, PALESTINE.

E-mail: hmdalloul@yahoo.com

\begin{abstract}
A series of 1,2,4,5-tetraaza-3-pentenes 4a-j were synthesized by the reaction of appropriate nitrilimines 2 with substituted hydrazines $\left(H_{2} N N H C O R, R=P h\right.$, OMe) 3. Heating the compounds $4 a-j$ with activated charcoal in refluxing benzene oxidized to formazans $5 a-j$ and some formazans $5 f, j(R=O M e)$ gave s-tetrazinones $6 f, j$ in presence of lithium hydride. The microanalysis and spectral data of the synthesized compounds are in full agreement with their molecular structure.
\end{abstract}

\section{KEYWORDS}

Nitrilimines, 1,2,4,5-tetraaza-3-pentenes, formazans, s-tetrazinones

\section{RESUMO}

Uma série de 1,2,4,5-tetraazo -3-pentenos 4a-j foram sintetizados pela reação das nitriliminas apropriadas 2 com hidrazinas substituidas $\left(H_{2} N N H C O R, R=P h, O M e\right) 3$. $O$ aquecimento dos compostos $4 a-j$ com carvão ativado em benzeno quente levou á oxidação para formazanaos $5 a j$ e alguns formazanos $5 f j(R=O M e)$ e na presença de LiH formaram s-tetrazinonas $6 f j$. As microanalises o os dados espectrais concordam com as estruturas moleculares dos compostos.

\section{PALAVRAS CHAVE:}

Nitriliminas, 1,2,4,5 tetraazo-3-pentenos, formazanas, s-tetrazinonas 


\section{GRAPHICAL ABSTRACT}

Reaction of Nitrilimines with Substituted Hydrazines:

Synthesis of 1,2,4,5-Tetraaza-3-pentenes and Formazans HANY M. DALLOUL

Alaqsa University of Gaza, Palestine.

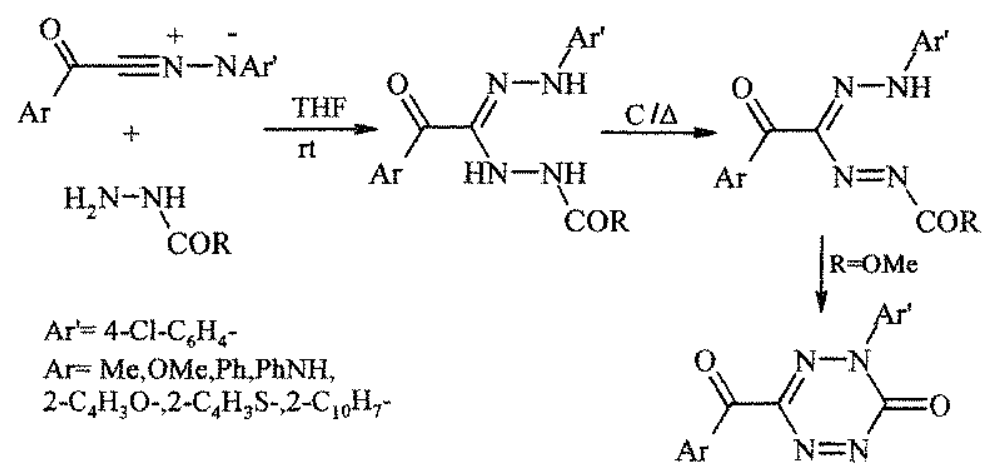

VISIT OUR SITE: http://www.sbjchem.he.com.br 


\section{INTRODUCTION}

The preparation of hydrazonoyl halides is well known because of their extensive use in 1,3-dipolar cycloaddition and cyclocondensation reactions. El-Haddad et al. [1] was reported the synthesis of 1-methoxycarbonyl-2-[1-(4-chlorophenyl)hydrazonopropan-2-one]hydrazine, which oxidized upon heating with charcoal in refluxing toluene to 3-acetyl-1-methoxy-carbonyl-5-(4-chlorophenyl)formazan.

The reactions nitrilimines and nitrile oxides were recently reviewed by Ferwanah et al. [2]. In a continuation of our work concerning the utility of nitrilimines in the synthesis of aza compounds, we investigated the reaction of different $\mathrm{C}$-substituted-Narylnitrilimines with benzoyl- and methoxycarbonyl hydrazines.

\section{RESULTS AND DISCUSSION}

The precursors of nitrilimines hydrazonoyl chlorides I employed in this study were prepared according to reported literature procedures [3-7]. The non isolable nitrilimines II immediately reacted with benzoyl and methoxycarbonyl hydrazines III affording the corresponding acyclic adducts, 1,2,4,5-tetraaza-3-pentenes IVa-j (Scheme 1) in good yields. Structural assignment of IVa-j was based on elemental analysis and spectral data. IR spectra of these compounds revealed the presence of the characteristic functional groups. The signals of the $\mathrm{OCH}_{3}$ in both ${ }^{1} \mathrm{H}$ - and ${ }^{13} \mathrm{C}$ NMR spectra is of particular importance in support of the suggested acyclic structure. The spectral data of the obtained compounds IV a-j are presented in the experimental section.

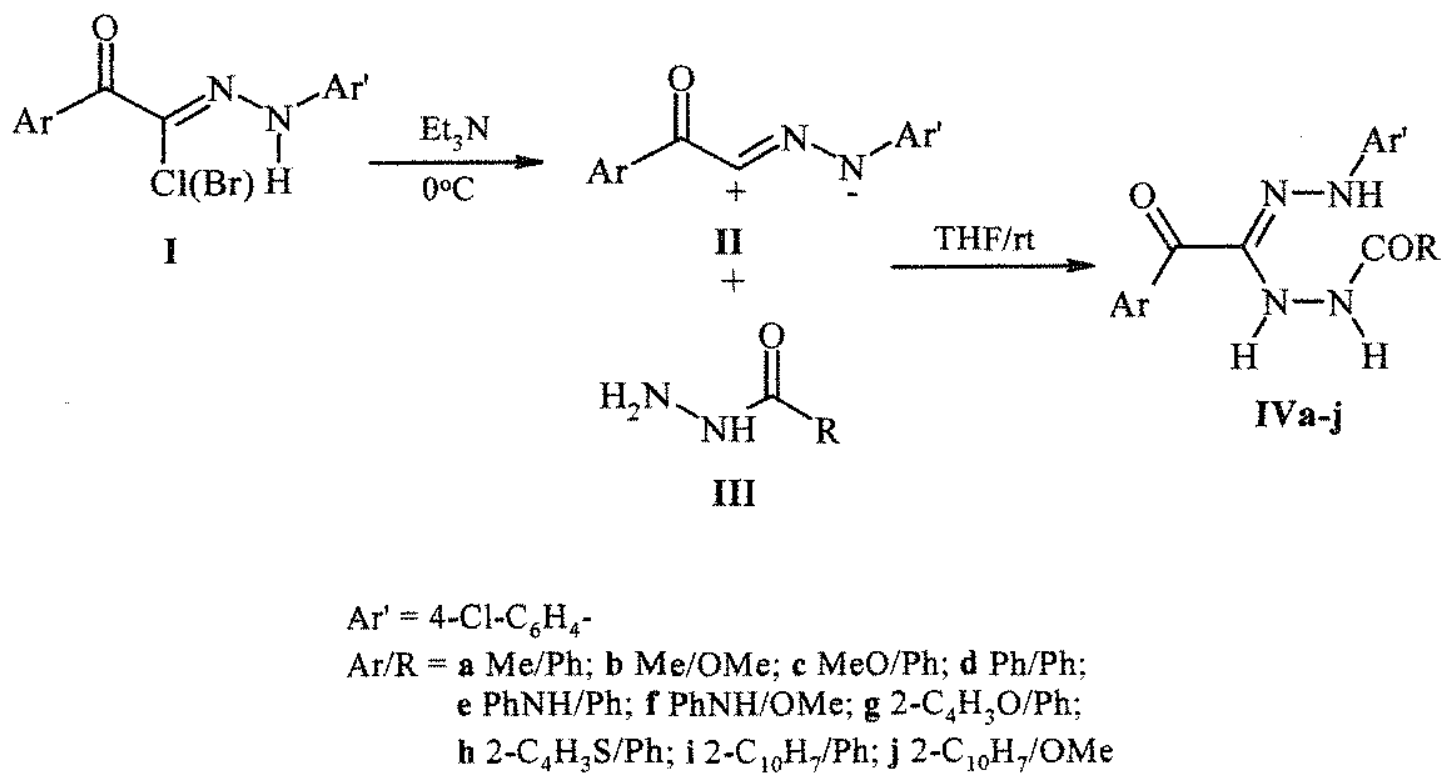

Figure 1. Synthetic pathway for the preparation of compounds IVa-j. 
The acyclic adducts Va-j were oxidized to the corresponding formazans $\mathbf{V a - j}$ by heating them with activated charcoal in refluxing benzene or toluene for 6 hours (Scheme 2). No other cyclic products were observed using TLC. Structure elucidation of the obtained formazans Va-j was achieved by analytical and spectral data summarized in the experimental section. Their IR spectra revealed the absence of two $\mathrm{NH}$ absorption bands. Both and ${ }^{1} \mathrm{H}-$ and ${ }^{13} \mathrm{C}$ NMR spectra of compounds Va-j showed all the signals of the proposed structures, indicating the disappearance of $\mathrm{HN}-\mathrm{NH}$ protons, however, the $\mathrm{OCH}_{3}$ signal in compounds $\mathbf{V b}, \mathbf{f}, \mathbf{j}$ does not disappeared which indicated that those compounds are oxidized to the formazans without further cyclization to the expected tetrazinones.

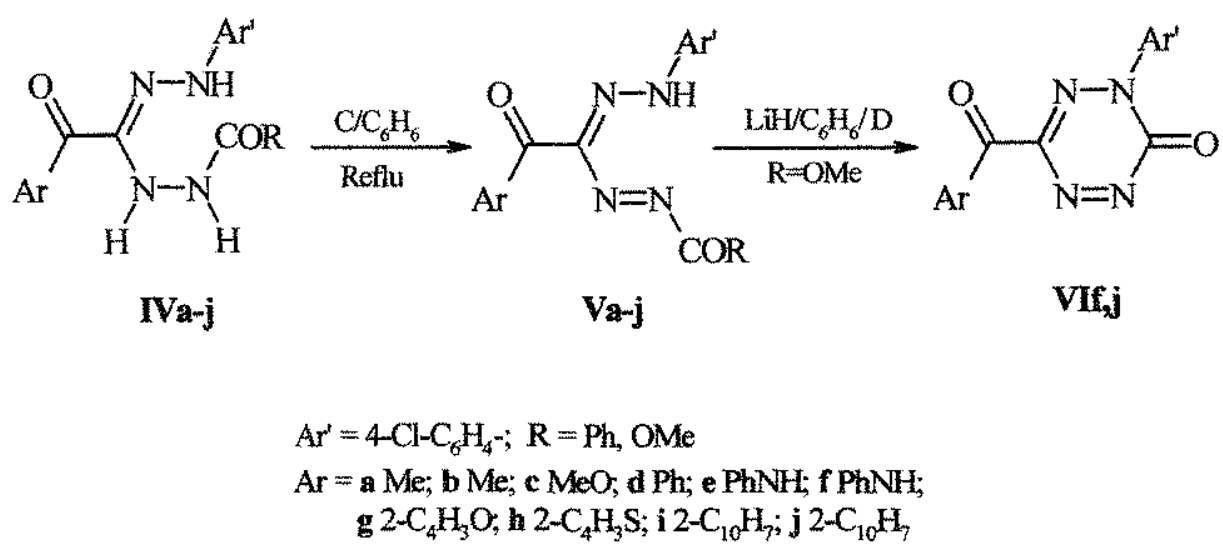

Figure 2. Synthetic pathway for the preparation of compounds Va-j and VIf,j.

The thermal cyclization of formazans $\mathbf{V} \mathbf{f}, \mathbf{j}$ was performed by heating them with lithium hydride in benzene for 4 hours. New products were formed as indicated by TLC and found to be s-tetrazinones VIf,j (Figure 2). Structural assignment of compounds VIf,j is based on elemental analysis, mass spectra and NMR results. Elemental analysis and mass spectra showed a loss of methanol molecule. Further evidence was obtained from NMR measurements. The ${ }^{1} \mathrm{H}$ NMR indicate that the $\mathrm{NH}(\delta=11.40 \mathrm{ppm})$ and methoxy protons $(\delta=3.90 \mathrm{ppm})$ are disappeared. Also the ${ }^{13} \mathrm{C}$ NMR data illustrated that compounds VIf, $\mathbf{j}$ have the assigned cyclic structure by the absence of signal for methoxy carbon $(\delta=53.80 \mathrm{ppm})$ and the presence of the signal at $\delta=159 \mathrm{ppm}$ for the carbonyl carbon of tetrazinone ring. 


\section{EXPERIMENTAL SECTION}

\subsection{Reagents and Instrumentation}

Melting points were determined on an A. Krüss Melting Point Meter equipped with a thermometer and are uncorrected. The IR spectra were measured as potassium bromide pellets using a Satellite 3000 Mid infrared spectrophotometer. The ${ }^{1} \mathrm{H}$ NMR and ${ }^{13} \mathrm{C}$ NMR spectra were recorded on a Bruker AM $300 \mathrm{MHz}$ spectrometer at room temperature in DMSO- $\mathrm{d}_{6}$ solution using tetramethylsilane (TMS) as internal reference. Chemical shifts were recorded as $\delta$ values in parts per millions (ppm) downfield from internal TMS. Electron impact (EI) mass spectra were run on a Shimadzu GCMSQP1000 EX spectrometer at $70 \mathrm{eV}$. All compounds were analyzed satisfactorily for $\mathrm{C}$, $\mathrm{H}$ and $\mathrm{N}$. The hydrazonoyl halides Ia-j $[3-7]$ were prepared according to literature procedures. Tetrahydrofuran (THF) and triethylamine were obtained from Across Company, Belgium. Benzoic acid hydrazide and methyl hydrazinocarboxylate were purchased from Avocado Research Chemicals, England, and used without further purification.

\subsection{Synthesis of Compounds IVa-j}

To a stirred solution of the appropriate hydrazonoyl halide I (10 $\mathrm{mmol})$ and hydrazine III $(20 \mathrm{mmol})$ in THF $(70 \mathrm{~mL})$, triethylamine $(4 \mathrm{~mL}, 30 \mathrm{mmol})$ in THF (10 $\mathrm{mL}$ ) was dropwise added at $0{ }^{\circ} \mathrm{C}$. The temperature of the reaction mixture was then allowed to rise slowly to room temperature, and stirring was continued overnight. The solvent was then evaporated in vacuo, and the residue washed with water $(100 \mathrm{~mL})$. The resulting crude solid product was collected and recrystallized from ethanol. The following compounds were prepared using this method:

3-Acetyl-1-benzoyl-5-(4-chlorophenyl)-1,2,4,5-tetraaza-3-pentene (IVa); Yield: $75 \%$; m.p.: $177-179^{\circ} \mathrm{C}$; IR (KBr) $\left.v_{\max }: \mathrm{cm}^{-1} 3432,3342,3315 \mathrm{NH}\right), 1693\left(\mathrm{CH}_{3}-\right.$ $\mathrm{C}=\mathrm{O}) 1675(\mathrm{Ph}-\mathrm{C}=\mathrm{O}), 1592(\mathrm{C}=\mathrm{N}) ;{ }^{1} \mathrm{H}$ NMR $\left(\mathrm{CDCl}_{3}\right): \delta 9.50(s, 1 \mathrm{H}, \mathrm{NH}), 8.90(s$, $1 \mathrm{H}, \mathrm{NH}), 8.27-7.26(\mathrm{~m}, 9 \mathrm{H}, \mathrm{Ar}-\mathrm{H}), 7.31(\mathrm{~s}, 1 \mathrm{H}, \mathrm{NH}), 2.45\left(s, 3 \mathrm{H}, \mathrm{COCH}_{3}\right) ;{ }^{13} \mathrm{C} \mathrm{NMR}$ $\left(\mathrm{CDCl}_{3}\right): \delta 192.3,169.1,141.0,139.6,135.6,133.2,130.8,130.4,129.3,128.5,120.4$, 24.5; MS: $m / z$ 330/332 [M" $\mathrm{M}^{+}$, Chlorine isotopes]; Anal. Calcd. for $\mathrm{C}_{16} \mathrm{H}_{15} \mathrm{~N}_{4} \mathrm{O}_{2}$ (330.78): C, $58.10 ; \mathrm{H}, 4.57 ; \mathrm{N}, 16.94$; Found: C, $57.85 ; \mathrm{H}, 4.45 ; \mathrm{N}, 17.10$.

3-Acetyl-5-(4-chlorophenyl)-1-methoxycarboyl-1,2,4,5-tetraaza-3-pentene

(IVb): Yield: 78\%; m.p.: 146-148 ${ }^{\circ} \mathrm{C}$; IR (KBr) $v_{\max }: \mathrm{cm}^{-1} 3434,3340,3319$ (NH), 1693 $\left(\mathrm{CH}_{3}-\mathrm{C}=\mathrm{O}\right) 1710(\mathrm{O}-\mathrm{C}=\mathrm{O}), 1596(\mathrm{C}=\mathrm{N}) ;{ }^{1} \mathrm{H}$ NMR $\left(\mathrm{CDCl}_{3}\right): \delta 9.54(s, 1 \mathrm{H}, \mathrm{NH}), 8.86$ $(s, 1 \mathrm{H}, \mathrm{NH}), 7.45-6.86(m, 4 \mathrm{H}, \mathrm{Ar}-\mathrm{H}), 7.36(s, 1 \mathrm{H}, \mathrm{NH}), 3.61\left(s, 3 \mathrm{H}, \mathrm{OCH}_{3}\right), 2.42(s$, $\left.3 \mathrm{H}, \mathrm{COCH}_{3}\right) ;{ }^{13} \mathrm{C} \mathrm{NMR}\left(\mathrm{CDCl}_{3}\right): \delta 192.1,156.9,141.0,139.1,129.0,128.2,120.6$, 52.3, 24.6; MS: $m / z$ 284/286 [M ${ }^{+}$, Chlorine isotopes]; Anal. Calcd. for $\mathrm{C}_{11} \mathrm{H}_{13} \mathrm{~N}_{4} \mathrm{O}_{3}$ (284.70): C, 46.41; H, 4.60; N, 19.68; Found: C, 46.20; H, 4.72; N, 19.55. 


\section{SOUTH. BRAZ. J. CHEM., Vol.19, No. 19, 2011}

\section{Synthesis of 1,2,4,5-Tertaaza-3-Pentenes and Formazans}

1-Benzoyl-5-(4-chlorophenyl)-3-methoxycarbonyl-1,2,4,5-tetraaza-3-pentene (IVc): Yield: 76\%; m.p.: $169-171^{\circ} \mathrm{C}$; IR (KBr) v $v_{\max }: \mathrm{cm}^{-1} 3429,3337,3320(\mathrm{NH}), 1715$ $(\mathrm{O}-\mathrm{C}=\mathrm{O}) 1675(\mathrm{Ph}-\mathrm{C}=\mathrm{O}), 1594(\mathrm{C}=\mathrm{N}) ;{ }^{1} \mathrm{H}$ NMR $\left(\mathrm{CDCl}_{3}\right): \delta 9.56(s, 1 \mathrm{H}, \mathrm{NH}), 8.89(s$, $1 \mathrm{H}, \mathrm{NH}), 8.22-7.21(\mathrm{~m}, 9 \mathrm{H}, \mathrm{Ar}-\mathrm{H}), 7.38(s, 1 \mathrm{H}, \mathrm{NH}), 3.59\left(s, 3 \mathrm{H}, \mathrm{OCH}_{3}\right) ;{ }^{13} \mathrm{C} \mathrm{NMR}$ $\left(\mathrm{CDCl}_{3}\right): \delta 169.3,157.1,141.3,139.7,135.3,133.0,130.9,130.2,129.1,128.7,120.7$, 52.6; MS: $m / z$ 346/348 [M+ Chlorine isotopes]; Anal. Calcd. for $\mathrm{C}_{16} \mathrm{H}_{15} \mathrm{~N}_{4} \mathrm{O}_{3}$ (346.78): C, $55.42 ; \mathrm{H}, 4.36 ; \mathrm{N}, 16.16$; Found: $\mathrm{C}, 55.70 ; \mathrm{H}, 4.21 ; \mathrm{N}, 15.05$.

5-(4-Chlorophenyl)-1,3-dibenzoyl-1,2,4,5-tetraaza-3-pentene (IVd): Yield: $75 \%$; m.p.: $182-184^{\circ} \mathrm{C}$; IR (KBr) $v_{\max }: \mathrm{cm}^{-1} 3427,3337,3322(\mathrm{NH}), 1677(\mathrm{~N}-\mathrm{C}=\mathrm{O})$, $1640(\mathrm{Ph}-\mathrm{C}=\mathrm{O}), 1598(\mathrm{C}=\mathrm{N}) ;{ }^{1} \mathrm{H}$ NMR $\left(\mathrm{CDCl}_{3}\right): \delta 10.31(s, 1 \mathrm{H}, \mathrm{NH}), 8.31(s, 1 \mathrm{H}, \mathrm{NH})$, 8.17-7.10 $(m, 14 \mathrm{H}, \mathrm{Ar}-\mathrm{H}), 7.37(s, 1 \mathrm{H}, \mathrm{NH}) ;{ }^{13} \mathrm{C} \mathrm{NMR}\left(\mathrm{CDCl}_{3}\right): \delta 187.5,169.1,141.7$, $139.5,136.4,135.6,133.2,132.2,130.7,130.1,129.3,128.5,127.9,127.1,115.8$; MS: $m / z$ 392/394 [M $\mathrm{M}^{+}$, Chlorine isotopes]; Anal. Calcd. for $\mathrm{C}_{21} \mathrm{H}_{17} \mathrm{ClN}_{4} \mathrm{O}_{2}$ (392.85): $\mathrm{C}$, 64.21; H, 4.36; N, 14.26; Found: C, 63.98; H, 4.50; N, 14.37 .

1-Benzoyl-5-(4-chlorophenyl)-3-phenylaminocarbonyl-1,2,4,5-tetraaza-3-

pentene (IVe): Yield: $77 \%$; m.p.: $178-180^{\circ} \mathrm{C}$; IR (KBr) $v_{\max }: \mathrm{cm}^{-1} 3435,3340,3328$, $3275(\mathrm{NH}), 1675(\mathrm{Ph}-\mathrm{C}=\mathrm{O}), 1655($ amide $\mathrm{C}=\mathrm{O}), 1605(\mathrm{C}=\mathrm{N}) ;{ }^{1} \mathrm{H}$ NMR $\left(\mathrm{CDCl}_{3}\right): \delta$ $10.36,(\mathrm{~s}, 1 \mathrm{H}, \mathrm{NH}), 9.50(s, 1 \mathrm{H}, \mathrm{NH}), 8.92(s, 1 \mathrm{H}, \mathrm{NH}), 8.83(s, 1 \mathrm{H}, \mathrm{NH}), 8.27-7.26(m$, $14 \mathrm{H}, \mathrm{Ar}-\mathrm{H}), 7.31(s, 1 \mathrm{H}, \mathrm{NH}) ;{ }^{13} \mathrm{C}$ NMR $\left(\mathrm{CDCl}_{3}\right): \delta 169.3,160.3,142.5,138.6,135.6$, $133.2,130.8,130.4,129.4,128.7,128.5,125.0,123.6,120.5,116.2$; MS: $m / z$ 407/409 $\left[\mathrm{M}^{+}\right.$, Chlorine isotopes]; Anal. Calcd. for $\mathrm{C}_{21} \mathrm{H}_{18} \mathrm{ClN}_{5} \mathrm{O}_{2}(407.86): \mathrm{C}, 61.84 ; \mathrm{H}, 4.45 ; \mathrm{N}$, 17.17; Found: $\mathrm{C}, 62.05 ; \mathrm{H}, 4.30 ; \mathrm{N}, 17.00$.

5-(4-Chlorophenyl)-1-methoxycarboyl-3-phenylaminocarbonyl-1,2,4,5-tetraaza-3-pentene(IVf): Yield: $74 \%$; m.p.: $158-160^{\circ} \mathrm{C}$; IR (KBr) $v_{\max }: \mathrm{cm}^{-1} 3433,3335$, $3325,3270(\mathrm{NH}), 1725(\mathrm{O}-\mathrm{C}=\mathrm{O}), 1653($ amide $\mathrm{C}=\mathrm{O}), 1600(\mathrm{C}=\mathrm{N}) ;{ }^{1} \mathrm{H} \mathrm{NMR}\left(\mathrm{CDCl}_{3}\right)$ : $\delta 9.49(s, 1 \mathrm{H}, \mathrm{NH}), 8.91(s, 1 \mathrm{H}, \mathrm{NH}), 8.82(s, 1 \mathrm{H}, \mathrm{NH}), 7.45-6.86(m, 9 \mathrm{H}, \mathrm{Ar}-\mathrm{H}), 7.36$ $(s, 1 \mathrm{H}, \mathrm{NH}), 3.61\left(s, 3 \mathrm{H}, \mathrm{OCH}_{3}\right) ;{ }^{13} \mathrm{C} \mathrm{NMR}\left(\mathrm{CDCl}_{3}\right): \delta 160.2,157.6,142.0,138.1$, $135.2,129.7,129.0,128.2,127.3,125.1,119.8,52.3 ; \mathrm{MS}: \mathrm{m} / z$ 361/363 [M+ Chlorine isotopes]; Anal. Calcd. for $\mathrm{C}_{16} \mathrm{H}_{16} \mathrm{CIN}_{5} \mathrm{O}_{3}(361.79)$ : $\mathrm{C}, 53.12 ; \mathrm{H}, 4.46 ; \mathrm{N}, 19.36$; Found: C, $52.86 ; \mathrm{H}, 4.35 ; \mathrm{N}, 19.45$.

1-Benzoyl-5-(4-chlorophenyl)-3-(2-furoyl)-1,2,4,5-tetraaza-3-pentene (IVg): Yield: $73 \%$; m.p.: $166-168^{\circ} \mathrm{C}$; IR (KBr) v $v_{\max }: \mathrm{cm}^{-1} 3433,3341,3321(\mathrm{NH}), 1676(\mathrm{Ph}-$ $\mathrm{C}=\mathrm{O}), 1665(\mathrm{C}=\mathrm{O}), 1597(\mathrm{C}=\mathrm{N})$; ${ }^{\mathrm{l}} \mathrm{H} \mathrm{NMR}\left(\mathrm{CDCl}_{3}\right): \delta 9.49(s, 1 \mathrm{H}, \mathrm{NH}), 8.89(s, 1 \mathrm{H}$, $\mathrm{NH}), 8.21-7.13(m, 12 \mathrm{H}, \mathrm{Ar}-\mathrm{H}), 7.33(s, 1 \mathrm{H}, \mathrm{NH}) ;{ }^{13} \mathrm{C} \mathrm{NMR}\left(\mathrm{CDCl}_{3}\right): \delta 174.2,169.2$, $141.6,140.1,139.1,135.6,135.1,133.2,130.8,130.6,129.0,128.1,127.9,125.4$, 120.6; MS: $m / z$ 382/384 [M ${ }^{+}$; Anal. Calcd. for $\mathrm{C}_{19} \mathrm{H}_{15} \mathrm{ClN}_{4} \mathrm{O}_{3}(382.81): \mathrm{C}, 59.62 ; \mathrm{H}$, 3.95 ; N, 14.64; Found: C, 59.40; H, 4.10; N, 14.55 .

1-Benzoyl-5-(4-chlorophenyl)-3-(2-thenoyl)-1,2,4,5-tetraaza-3-pentene (IVh): Yield: $75 \%$; m.p.: $159-161^{\circ} \mathrm{C}$; IR (KBr) $v_{\max }: \mathrm{cm}^{-1} 3436,3340,3325(\mathrm{NH}), 1678$ ( Ph$\mathrm{C}=\mathrm{O}), 1660(\mathrm{C}=\mathrm{O}), 1596(\mathrm{C}=\mathrm{N}) ;{ }^{1} \mathrm{H} \mathrm{NMR}\left(\mathrm{CDCl}_{3}\right): \delta 9.46(s, 1 \mathrm{H}, \mathrm{NH}), 8.87(s, 1 \mathrm{H}$, $\mathrm{NH}), 8.30-7.10(m, 12 \mathrm{H}, \mathrm{Ar}-\mathrm{H}), 7.31(s, 1 \mathrm{H}, \mathrm{NH}) ;{ }^{13} \mathrm{C} \mathrm{NMR}\left(\mathrm{CDCl}_{3}\right): \delta 174.6,169.3$, $141.3,140.2,139.3,135.1,134.9,133.2,130.8,130.4,129.3,128.3,128.0,125.3$, 120.4; MS: $\mathrm{m} / z$ 398/400 $\left[\mathrm{M}^{+}\right.$, Chlorine isotopes]; Anal. Calcd. for $\mathrm{C}_{19} \mathrm{H}_{15} \mathrm{ClN}_{4} \mathrm{O}_{2} \mathrm{~S}$ (334.38): C, 57.21; H, 3.79; N, 14.05; Found: C, 57.45; H, 3.90; N, 13.95. 
1-Benzoyl-5-(4-chlorophenyl)-3-(2-naphthoyl)-1,2,4,5-tetraaza-3-pentene (IVi): Yield: $71 \%$; m.p.: $171-173^{\circ} \mathrm{C}$; IR (KBr) v $\max : \mathrm{cm}^{-1} 3424,3331,3309(\mathrm{NH}), 1693\left(\mathrm{CH}_{3}-\right.$ $\mathrm{C}=\mathrm{O}) 1675(\mathrm{Ph}-\mathrm{C}=\mathrm{O}), 1592(\mathrm{C}=\mathrm{N}) ;{ }^{l} \mathrm{H} \mathrm{NMR}\left(\mathrm{CDCl}_{3}\right): \delta 9.50(s, 1 \mathrm{H}, \mathrm{NH}), 8.90(s$, $1 \mathrm{H}, \mathrm{NH}), 8.77-7.26(m, 16 \mathrm{H}, \mathrm{Ar}-\mathrm{H}), 7.36(s, 1 \mathrm{H}, \mathrm{NH}) ;{ }^{13} \mathrm{C} \mathrm{NMR}\left(\mathrm{CDCl}_{3}\right): \delta 183.3$, $169.2,141.4,139.6,135.9,133.8,133.1,132.4,132.2,130.3,129.9,129.1,128.7$, $128.6,127.9,127.8,127.7,127.4,126.6,125.5,124.3 ; \mathrm{MS}: \mathrm{m} / z$ 442/444 [M+ Chlorine isotopes]; Anal. Calcd, for $\mathrm{C}_{25} \mathrm{H}_{19} \mathrm{CNN}_{4} \mathrm{O}_{2}(442.91): \mathrm{C}, 67.80 ; \mathrm{H}, 4.32 ; \mathrm{N}, 12.65$; Found: $\mathrm{C}, 68.05 ; \mathrm{H}, 4.20 ; \mathrm{N}, 12.50$.

5-(4-Chlorophenyl)-1-methoxycarbonyl-3-(2-naphthoyl)-1,2,4,5-tetraaza-3pentene (IVj): Yield: 74\%; m.p.: 179-181 ${ }^{\circ} \mathrm{C}$; IR (KBr) $v_{\max }: \mathrm{cm}^{-1} 3430,3333,3311$ $(\mathrm{NH}), 1693\left(\mathrm{CH}_{3} \mathrm{C}=\mathrm{O}\right) 1675(\mathrm{O}-\mathrm{C}=\mathrm{O}), 1592(\mathrm{C}=\mathrm{N}) ;{ }^{1} \mathrm{H}$ NMR $\left(\mathrm{CDCl}_{3}\right): \delta 9.49(s, 1 \mathrm{H}$, $\mathrm{NH}), 8.86(s, 1 \mathrm{H}, \mathrm{NH}), 8.75-7.24(m, 11 \mathrm{H}, \mathrm{Ar}-\mathrm{H}), 7.32(s, 1 \mathrm{H}, \mathrm{NH}), 3.57(s, 3 \mathrm{H}$, $\left.\mathrm{OCH}_{3}\right) ;{ }^{13} \mathrm{C} \mathrm{NMR}\left(\mathrm{CDCl}_{3}\right): \delta 183.6,157.8,141.4,139.1,135.6,134.1,132.5,132.3$, $130.5,129.9,129.2,128.4,127.8,127.7,126.5,125.6,124.4,53.1 ; \mathrm{MS}: m / z 396 / 398$ $\left[\mathrm{M}^{+}\right.$, Chlorine isotopes]; Anal. Calcd. for $\mathrm{C}_{20} \mathrm{H}_{19} \mathrm{ClN}_{4} \mathrm{O}_{3}(396.84): \mathrm{C}, 60.53 ; \mathrm{H}, 4.32 ; \mathrm{N}$, 14.12; Found: $\mathrm{C}, 60.35 ; \mathrm{H}, 4.40 ; \mathrm{N}, 14.05$.

\subsection{Synthesis of formazans Va-j}

Compounds IVa-j $(5 \mathrm{mml})$ were refluxed in benzene or toluene $(50 \mathrm{~mL})$ and activated charcoal $(1.0 \mathrm{~g})$ for 6 hours. After cooling the reaction was then filtered and the solvent was removed under reduced pressure and the residual solid was collected and recrystallized from chloroform/petroleum ether (b.p. 40-60 oC) to give formazans Va-j. The following compounds were prepared using this method:

3-Acetyl-1-benzoyl-5-(4-chlorophenyl)formazan (Va): Yield: 76\%; m.p.: 149$151{ }^{\circ} \mathrm{C}$; IR ( $\left.\mathrm{KBr}\right) v_{\max }: \mathrm{cm}^{-1} 3348(\mathrm{NH}), 1685\left(\mathrm{CH}_{3}-\mathrm{C}=\mathrm{O}\right) 1679(\mathrm{Ph}-\mathrm{C}=\mathrm{O}), 1590$ $(\mathrm{C}=\mathrm{N}) ;{ }^{1} \mathrm{H}$ NMR $\left(\mathrm{CDCl}_{3}\right): \delta 11.49(s, 1 \mathrm{H}, \mathrm{NH}), 7.40-7.29(m, 11 \mathrm{H}, \mathrm{Ar}-\mathrm{H}), 2.60(s, 3 \mathrm{H}$, $\left.\mathrm{COCH}_{3}\right) ;{ }^{13} \mathrm{C} \mathrm{NMR}\left(\mathrm{CDCl}_{3}\right): \delta 193.9\left(\mathrm{CH}_{3}-\mathrm{C}=\mathrm{O}\right), 169.1(\mathrm{~N}-\mathrm{C}=\mathrm{O}), 150.5(\mathrm{C}=\mathrm{N}), 26.6$ $\left(\mathrm{COCH}_{3}\right) ; \mathrm{MS}: \mathrm{m} / z$ 328/330 [M+ Chlorine isotopes]; Anal. Calcd. for $\mathrm{C}_{16} \mathrm{H}_{13} \mathrm{ClN}_{4} \mathrm{O}_{2}$ (328.76): C, 58.46; H, 3.99; N, 17.04; Found: C, 58.25; H, 4.10; N, 16.90 .

3-Acetyl-5-(4-chlorophenyl)-1-methoxycarboylformazan (Vb): Yield: 79\%; m.p.: $136-138^{\circ} \mathrm{C}$; IR (KBr) $v_{\max }: \mathrm{cm}^{-1} 3347(\mathrm{NH}), 1720(\mathrm{O}-\mathrm{C}=\mathrm{O}), 1686\left(\mathrm{CH}_{3}-\mathrm{C}=\mathrm{O}\right)$, $1590(\mathrm{C}=\mathrm{N}) ;{ }^{1} \mathrm{H}$ NMR $\left(\mathrm{CDCl}_{3}\right): \delta 11.51(s, 1 \mathrm{H}, \mathrm{NH}), 7.42-7.31(m, 11 \mathrm{H}, \mathrm{Ar}-\mathrm{H}), 3.91(s$, $\left.3 \mathrm{H}, \mathrm{OCH}_{3}\right), 2.61\left(\mathrm{~s}, 3 \mathrm{H}, \mathrm{COCH}_{3}\right) ;{ }^{13} \mathrm{C} \mathrm{NMR}\left(\mathrm{CDCl}_{3}\right): \delta 193.9\left(\mathrm{CH}_{3}-\mathrm{C}=\mathrm{O}\right), 153.8(\mathrm{~N}-$ $\mathrm{C}=\mathrm{O}), 150.4(\mathrm{C}=\mathrm{N}), 53.9\left(\mathrm{OCH}_{3}\right), 26.6\left(\mathrm{COCH}_{3}\right) ; \mathrm{MS}: \mathrm{m} / z 282 / 284\left[\mathrm{M}^{+}\right.$, Chlorine isotopes]; Anal. Calcd. for $\mathrm{C}_{11} \mathrm{H}_{11} \mathrm{ClN}_{4} \mathrm{O}_{3}(282.69)$ : $\mathrm{C}, 46.74 ; \mathrm{H}, 3.92 ; \mathrm{N}, 19.82$; Found: C, $46.60 ; \mathrm{H}, 4.05 ; \mathrm{N}, 19.70$.

1-Benzoyl-5-(4-chlorophenyl)-3-methoxycarbonylformazan (Vc): Yield: 80\%; m.p.: $127-129^{\circ} \mathrm{C}$; IR (KBr) $v_{\max }: \mathrm{cm}^{-1} 3325(\mathrm{NH}), 1725(\mathrm{O}-\mathrm{C}=\mathrm{O}), 1673(\mathrm{Ph}-\mathrm{C}=\mathrm{O})$, $1586(\mathrm{C}=\mathrm{N}) ;{ }^{1} \mathrm{H} \mathrm{NMR}\left(\mathrm{CDCl}_{3}\right): \delta 11.43(s, 1 \mathrm{H}, \mathrm{NH}), 7.45-7.21(m, 11 \mathrm{H}, \mathrm{Ar}-\mathrm{H}), 3.92(s$, $\left.3 \mathrm{H}, \mathrm{OCH}_{3}\right), 2.61\left(s, 3 \mathrm{H}, \mathrm{COCH}_{3}\right) ;{ }^{13} \mathrm{C} \mathrm{NMR}\left(\mathrm{CDCl}_{3}\right): \delta 168.8(\mathrm{~N}-\mathrm{C}=\mathrm{O}), 158.4(\mathrm{O}-$ $\mathrm{C}=\mathrm{O}), 149.7(\mathrm{C}=\mathrm{N}), 53.9\left(\mathrm{OCH}_{3}\right) ; \mathrm{MS}: \mathrm{m} / z$ 344/346 [M+, Chlorine isotopes]; Anal. Calcd. for $\mathrm{C}_{16} \mathrm{H}_{13} \mathrm{ClN}_{4} \mathrm{O}_{3}(344.76): \mathrm{C}, 55.74 ; \mathrm{H}, 3.80 ; \mathrm{N}, 16.25$; Found: C, 55.90; H, $3.72 ; \mathrm{N}, 16.33$. 


\section{SOUTH. BRAZ. J. CHEM., Vol.19, No. 19, 2011}

\section{Synthesis of 1,2,4,5-Teraaza-3-Pentenes and Formazans}

5-(4-Chlorophenyl)-1,3-dibenzoylformazan (Vd): Yield: 81\%; m.p.: 176-178 ${ }^{\circ} \mathrm{C}$; IR $(\mathrm{KBr}) v_{\max }: \mathrm{cm}^{-1} 3322(\mathrm{NH}), 1673(\mathrm{Ph}-\mathrm{C}=\mathrm{O}), 1645(\mathrm{Ar}-\mathrm{C}=\mathrm{O}), 1585(\mathrm{C}=\mathrm{N}) ;{ }^{1} \mathrm{H}$ $\mathrm{NMR}\left(\mathrm{CDCl}_{3}\right): \delta 11.43(s, 1 \mathrm{H}, \mathrm{NH}), 7.75-7.20(\mathrm{~m}, 11 \mathrm{H}, \mathrm{Ar}-\mathrm{H}) ;{ }^{13} \mathrm{C} \mathrm{NMR}\left(\mathrm{CDCl}_{3}\right): \delta$ $185.5(\mathrm{Ph}-\mathrm{C}=\mathrm{O}), 168.7(\mathrm{~N}-\mathrm{C}=\mathrm{O}) 149.2(\mathrm{C}=\mathrm{N}) ; \mathrm{MS}: \mathrm{m} / \mathrm{z} 390 / 392 \quad\left[\mathrm{M}^{+}\right.$, Chlorine isotopes]; Anal. Calcd. for $\mathrm{C}_{21} \mathrm{H}_{15} \mathrm{ClN}_{4} \mathrm{O}_{2}(390.83)$ : $\mathrm{C}, 64.54 ; \mathrm{H}, 3.87 ; \mathrm{N}, 14.34$; Found: $\mathrm{C}, 64.40 ; \mathrm{H}, 3.75 ; \mathrm{N}, 14.50$.

1-Benzoyl-5-(4-chlorophenyl)-3-phenylaminocarbonyl formazan (Ve): Yield: 76\%; m.p.: $198-200^{\circ} \mathrm{C}$; IR ( $\left.\mathrm{KBr}\right) v_{\max }: \mathrm{cm}^{-1} 3328,3245(\mathrm{NH}), 1675(\mathrm{Ph}-\mathrm{C}=\mathrm{O}), 1655$ $(\mathrm{Ar}-\mathrm{C}=\mathrm{O}), 1592(\mathrm{C}=\mathrm{N}) ;{ }^{1} \mathrm{H}$ NMR $\left(\mathrm{CDCl}_{3}\right): \delta 11.36,(\mathrm{~s}, 1 \mathrm{H}, \mathrm{NH}), 8.92(s, 1 \mathrm{H}, \mathrm{NH})$, 8.31-7.26 $(m, 14 \mathrm{H}, \mathrm{Ar}-\mathrm{H}) ;{ }^{13} \mathrm{C}$ NMR $\left(\mathrm{CDCl}_{3}\right): \delta 168.3(\mathrm{~N}-\mathrm{C}=\mathrm{O}), 161.4(\mathrm{Ar}-\mathrm{C}=\mathrm{O})$, $150.3(\mathrm{C}=\mathrm{N})$; MS: $m / z$ 405/407 $\left[\mathrm{M}^{+}\right.$, Chlorine isotopes]; Anal. Calcd. for $\mathrm{C}_{21} \mathrm{H}_{16} \mathrm{ClN}_{5} \mathrm{O}_{2}$ (405.85): C, 62.15; H, 3.97; N, 17.26; Found: C, 61.95; H, 4.10; N, 17.35.

\section{5-(4-Chlorophenyl)-1-methoxycarboyl-3-phenylaminocarbonylformazan}

(Vf): Yield: $79 \%$; m.p.: $188-190^{\circ} \mathrm{C}$; IR (KBr) $v_{\max }: \mathrm{cm}^{-1} 3325,3236$ (NH), 1722 ( O$\mathrm{C}=\mathrm{O}), 1650(\mathrm{Ar}-\mathrm{C}=\mathrm{O}), 1590(\mathrm{C}=\mathrm{N}) ;{ }^{1} \mathrm{H}$ NMR $\left(\mathrm{CDCl}_{3}\right): \delta 11.39(s, 1 \mathrm{H}, \mathrm{NH}), 8.96(s$, $1 \mathrm{H}, \mathrm{NH}), 7.45-7.18(\mathrm{~m}, 9 \mathrm{H}, \mathrm{Ar}-\mathrm{H}), 3.90\left(s, 3 \mathrm{H}, \mathrm{OCH}_{3}\right) ;{ }^{13} \mathrm{C} \mathrm{NMR}\left(\mathrm{CDCl}_{3}\right): \delta 161.2$ $(\mathrm{Ar}-\mathrm{C}=\mathrm{O}), 158.6(\mathrm{O}-\mathrm{C}=\mathrm{O}), 150.1(\mathrm{C}=\mathrm{N}), 53.8\left(\mathrm{OCH}_{3}\right) ; \mathrm{MS}: \mathrm{m} / \mathrm{z} 359 / 361\left[\mathrm{M}^{+}\right.$, Chlorine isotopes]; Anal. Calcd. for $\mathrm{C}_{16} \mathrm{H}_{14} \mathrm{ClN}_{5} \mathrm{O}_{3}$ (359.77): $\mathrm{C}, 53.42 ; \mathrm{H}, 3.92 ; \mathrm{N}, 19.47$; Found: C, $53.60 ; \mathrm{H}, 4.05 ; \mathrm{N}, 19.35$.

1-Benzoyl-5-(4-chlorophenyl)-3-(2-furoyl) formazan (Vg): Yield: 81\%; m.p.: $172-173{ }^{\circ} \mathrm{C}$; IR $(\mathrm{KBr}) v_{\max }: \mathrm{cm}^{-1} 3329(\mathrm{NH}), 1673(\mathrm{Ph}-\mathrm{C}=\mathrm{O}), 1660(\mathrm{Ar}-\mathrm{C}=\mathrm{O}), 1591$ $(\mathrm{C}=\mathrm{N}) ;{ }^{1} \mathrm{H}$ NMR $\left(\mathrm{CDCl}_{3}\right): \delta 11.36(s, 1 \mathrm{H}, \mathrm{NH}), 7.45-6.71(m, 11 \mathrm{H}, \mathrm{Ar}-\mathrm{H}) ;{ }^{13} \mathrm{C} \mathrm{NMR}$ $\left(\mathrm{CDCl}_{3}\right): \delta 173.4(\mathrm{Ar}-\mathrm{C}=\mathrm{O}), 168.9(\mathrm{~N}-\mathrm{C}=\mathrm{O}), 150.2(\mathrm{C}=\mathrm{N}) ; \mathrm{MS}: m / z \quad 380 / 382\left[\mathrm{M}^{+}\right.$, Chlorine isotopes]; Anal. Calcd. for $\mathrm{C}_{19} \mathrm{H}_{13} \mathrm{ClN}_{4} \mathrm{O}_{3}(380.79): \mathrm{C}, 59.93 ; \mathrm{H}, 3.44 ; \mathrm{N}$, 14.71 ; Found: C, $60.15 ; \mathrm{H}, 3.30$; N, 14.60.

1-Benzoyl-5-(4-chlorophenyl)-3-(2-thenoyl) formazan (Vh): Yield: 82\%; m.p.: $165-167^{\circ} \mathrm{C}$; IR (KBr) $v_{\max }: \mathrm{cm}^{-1} 3325(\mathrm{NH}), 1673(\mathrm{Ph}-\mathrm{C}=\mathrm{O}), 1665(\mathrm{Ar}-\mathrm{C}=\mathrm{O}), 1593$ $(\mathrm{C}=\mathrm{N}) ;{ }^{1} \mathrm{H}$ NMR $\left(\mathrm{CDCl}_{3}\right): \delta 11.37(s, 1 \mathrm{H}, \mathrm{NH}), 7.45-6.71(m, 11 \mathrm{H}, \mathrm{Ar}-\mathrm{H}) ;{ }^{13} \mathrm{C} \mathrm{NMR}$ $\left(\mathrm{CDCl}_{3}\right): \delta 174.6(\mathrm{Ar}-\mathrm{C}=\mathrm{O}), 168.7(\mathrm{~N}-\mathrm{C}=\mathrm{O}), 150.7(\mathrm{C}=\mathrm{N}) ; \mathrm{MS}: m / z 396 / 398\left[\mathrm{M}^{+}\right.$, Chlorine isotopes]; Anal. Calcd. for $\mathrm{C}_{19} \mathrm{H}_{13} \mathrm{ClN}_{4} \mathrm{O}_{2} \mathrm{~S}(334.38): \mathrm{C}, 57.50 ; \mathrm{H}, 3.30 ; \mathrm{N}$, 14.12; Found: C, 57.32; H, 3.42; N, 13.98 .

1-Benzoyl-5-(4-chlorophenyl)-3-(2-naphthoyl)formazan (Vi): Yield: 76\%; m.p.: $189-191^{\circ} \mathrm{C}$; IR $(\mathrm{KBr}) v_{\max }: \mathrm{cm}^{-1} 3326(\mathrm{NH}), 1672(\mathrm{Ph}-\mathrm{C}=\mathrm{O}), 1645(\mathrm{Ar}-\mathrm{C}=\mathrm{O})$, $1591(\mathrm{C}=\mathrm{N}) ;{ }^{1} \mathrm{H}$ NMR $\left(\mathrm{CDCl}_{3}\right): \delta 11.40(s, 1 \mathrm{H}, \mathrm{NH}), 8.63-7.49(m, 11 \mathrm{H}, \mathrm{Ar}-\mathrm{H}) ;{ }^{13} \mathrm{C}$ $\mathrm{NMR}\left(\mathrm{CDCl}_{3}\right): \delta 183.7(\mathrm{Ar}-\mathrm{C}=\mathrm{O}), 168.5(\mathrm{~N}-\mathrm{C}=\mathrm{O}), 149.9(\mathrm{C}=\mathrm{N}) ; \mathrm{MS}: m / z$ 440/442 $\mathrm{M}^{+}$, Chlorine isotopes]; Anal. Calcd. for $\mathrm{C}_{25} \mathrm{H}_{17} \mathrm{ClN}_{4} \mathrm{O}_{2}$ (440.89): C, 68.11; H, 3.89; N, 12.71; Found: C, 67.95; H, 4.00; N, 12.60 .

5-(4-Chlorophenyl)-1-methoxycarbonyl-3-(2-naphthoyl)formazan

(Vj): Yield: $78 \%$; m.p.: $176-178^{\circ} \mathrm{C}$; IR (KBr) $v_{\max }: \mathrm{cm}^{-1} 3326(\mathrm{NH}), 1671(\mathrm{Ph}-\mathrm{C}=\mathrm{O}), 1645($ $\mathrm{Ar}-\mathrm{C}=\mathrm{O}), 1591(\mathrm{C}=\mathrm{N}) ;{ }^{1} \mathrm{H} \mathrm{NMR}\left(\mathrm{CDCl}_{3}\right): \delta 11.43(s, 1 \mathrm{H}, \mathrm{NH}), 8.65-7.51(m, 11 \mathrm{H}, \mathrm{Ar}-$ $\mathrm{H}), 3.90\left(s, 3 \mathrm{H}, \mathrm{OCH}_{3}\right) ;{ }^{13} \mathrm{C}$ NMR $\left(\mathrm{CDCl}_{3}\right): \delta 183.7(\mathrm{Ar}-\mathrm{C}=\mathrm{O}), 153.9(\mathrm{~N}-\mathrm{C}=\mathrm{O}), 149.8$ $(\mathrm{C}=\mathrm{N}), 53.8\left(\mathrm{OCH}_{3}\right)$; MS: $m / z$ 394/396 [M+ Chlorine isotopes]; Anal. Calcd. for $\mathrm{C}_{20} \mathrm{H}_{15} \mathrm{ClN}_{4} \mathrm{O}_{3}$ (394.82): $\mathrm{C}, 60.84 ; \mathrm{H}, 3.83 ; \mathrm{N}, 14.19 ;$ Found: $\mathrm{C}, 60.65 ; \mathrm{H}, 3.72 ; \mathrm{N}$, 14.10 . 
H M. M. Dalloul

\subsection{Thermal cyclization of compounds $V(f, j)$ to s-tetrazinones VI(f,j)}

To a stirred solution of compounds $\mathbf{V f , j}(5 \mathrm{mmol})$ in benzene $(50 \mathrm{~mL})$ was carefully added lithium hydride $(0.08 \mathrm{~g}, 10 \mathrm{mmol})$ at $\mathrm{r}$. $\mathrm{t}$. The resulting reaction mixture was refluxed for 4 hours. After cooling excess lithium hydride was destroyed with some drops of glacial acetic acid. The solvent was evaporated under reduced pressure and the residue was washed with water and then triturated with ethanol $(10 \mathrm{~mL})$. The resulting solid product was collected and recrystallized from diethyl ether/petroleum ether (b.p. 40-60 oC) to give s-tetrazinones VIf,j. The following compounds were synthesized using this method:

1-(4-Chlorophenyl)-3-phenylaminocarbonyl-s-tetrazinone (VIf): Yield: 84\%; m.p.: $158-160^{\circ} \mathrm{C} ; \mathrm{IR}(\mathrm{KBr}) v_{\max }: \mathrm{cm}^{-1} 3276(\mathrm{NH}), 1655(\mathrm{Ar}-\mathrm{C}=\mathrm{O}), 1598(\mathrm{C}=\mathrm{N}) ;{ }^{1} \mathrm{H}$ NMR (DMSO-d 6 ): $\delta 8.86(s, 1 \mathrm{H}, \mathrm{NH}), 7.45-7.18(m, 9 \mathrm{H}, \mathrm{Ar}-\mathrm{H}) ;{ }^{13} \mathrm{C}$ NMR (DMSO-d 6 ): $\delta 159.8(\mathrm{C}=\mathrm{O}), 157.8(\mathrm{PhNH}-\mathrm{C}=\mathrm{O}), 139.8(\mathrm{C}=\mathrm{N}) ; \mathrm{MS}: \mathrm{m} / z$ 327/329 [M+ Chlorine isotopes]; Anal. Calcd. for $\mathrm{C}_{15} \mathrm{H}_{10} \mathrm{ClN}_{5} \mathrm{O}_{2}$ (327.73): $\mathrm{C}, 54.97 ; \mathrm{H}, 3.08 ; \mathrm{N}, 21.37$; Found: C, 55.15; H, 2.95; N, 21.45.

1-(4-Chlorophenyl)-3-(2-naphthoyl)-s-tetrazinone (VIj): Yield: 82\%; m.p.: $168-170^{\circ} \mathrm{C}$; IR $(\mathrm{KBr}) v_{\max }: \mathrm{cm}^{-1} 1645(\mathrm{Ar}-\mathrm{C}=\mathrm{O}), 1599(\mathrm{C}=\mathrm{N}) ;{ }^{1} \mathrm{H}$ NMR $\left(\mathrm{DMSO}-\mathrm{d}_{6}\right): \delta$ 8.60-7.48 $(m, 11 \mathrm{H}, \mathrm{Ar}-\mathrm{H}) ;{ }^{13} \mathrm{C}$ NMR $\left(\mathrm{DMSO}_{-} \mathrm{d}_{6}\right): \delta 184.3(\mathrm{Ar}-\mathrm{C}=\mathrm{O}), 159.7(\mathrm{C}=\mathrm{O})$, $140.3(\mathrm{C}=\mathrm{N})$; MS: $m / z$ 362/364 [M $\mathrm{M}^{+}$Chlorine isotopes]; Anal. Calcd. for $\mathrm{C}_{19} \mathrm{H}_{11} \mathrm{ClN}_{4} \mathrm{O}_{2}$ (362.78): C, 62.91; H, 3.06; N, 15.44; Found: C, 63.10; H, 2.98; N, 15.53.

\section{CONCLUSION}

The nitrilimines IIa-j reacted with benzoyl and methoxycarbonyl hydrazines III affording the 1,2,4,5-tetraaza-3-pentenes IVa-j, which underwent thermal oxidation to the corresponding formazans $\mathbf{V a - j}$. The treatment of formazans $\mathbf{V} \mathbf{f}, \mathbf{j}$ with lithium hydride in refluxing benzene gave s-tetrazinones VIf,j.

\section{REFERENCES}

[1] El-Haddad M. R.; Ferwanah A. E. S.; Awadallah A. M., J. Prakt. Chem. 340, 623 (1998).

[2] Ferwanah A. R. S.; Awadallah A. M., Molecules, 10, 492 (2005).

[3] El-Abadelah M. M.; Hussein A. Q.; Thaher B. A., Heterocycles, 32, 1879 (1991).

[4] Hassaneen H. M.; Shawali A. S.; Elwan N. M.; Abounada N. M., Org. Prep. Proced. Int., 24, 171 (1992).

[5] Frohberg P.; Drutkowski G.; Wagner C., Eur. J. Org. Chem., 1654 (2002).

[6] Farag A. M.; Algharib M. S., Org. Prep. Proced. Int., 20, 521 (1988).

[7] Shawali A. S.; Abdelhamid A. O., Bull. Chem. Soc. Jap., 49, 321 (1976).

The SOUTHERN BRAZILIAN JOURNAL OF CHEMISTRY (ISSN: 2674-6891; 0104-5431) is an open-access journal since 1993. Journal DOI: 10.48141/SBJCHEM. http://www.sbjchem.com

This text was introduced in this file in 2021 for compliance reasons.

(C) The Author(s)

OPEN ACCESS. This article is licensed under a Creative Commons Attribution 4.0 (CC BY 4.0) International License, which permits use, sharing , adaptation , distribution , and reproduction in any medium or format, as long as you give appropriate credit to the original author (s) and the source, provide a link to the Creative Commons license, and indicate if changes were made. The images or other third-party material in this article are included in the article's Creative Commons license unless indicated otherwise in a credit line to the material. If material is not included in the article's Creative Commons license and your intended use is not permitted by statutory regulation or exceeds the permitted use, you will need to obtain permission directly from the copyright holder. To view a copy of this license, visit http://creativecommons.org/licenses/by/4.0/. 